\title{
BMJ Open Cohort profile: the Australian Longitudinal Study of Adults with Autism (ALSAA)
}

\author{
Samuel Arnold (D) ,,2 Kitty-Rose Foley, ${ }^{2,3}$ Ye In (Jane) Hwang, ${ }^{1,2}$ \\ Amanda L Richdale, ${ }^{2,4}$ Mirko Uljarevic, ${ }^{2,4,5}$ Lauren P Lawson, ${ }^{2,4}$ Ru Ying Cai, ${ }^{2,4}$ \\ Torbjorn Falkmer, ${ }^{2,6}$ Marita Falkmer, ${ }^{2,6,7}$ Nick G Lennox, ${ }^{2,8}$ Anna Urbanowicz, ${ }^{2,8,9}$ \\ Julian Trollor (1) ${ }^{1,2}$
}

To cite: Arnold S, Foley K$\mathrm{R}$, Hwang $\mathrm{Yl}(\mathrm{Jane})$, et al. Cohort profile: the Australian Longitudinal Study of Adults with Autism (ALSAA). BMJ Open 2019;9:e030798. doi:10.1136/ bmjopen-2019-030798

- Prepublication history for this paper is available online. To view these files, please visit the journal online (http://dx.doi. org/10.1136/bmjopen-2019030798).

SA and K-RF are joint first authors.

Received 02 April 2019 Revised 30 October 2019 Accepted 31 October 2019

Check for updates

(C) Author(s) (or their employer(s)) 2019. Re-use permitted under CC BY-NC. No commercial re-use. See rights and permissions. Published by BMJ.

For numbered affiliations see end of article.

Correspondence to Professor Julian Trollor; J.Trollor@unsw.edu.au

\section{ABSTRACT}

Purpose There is a significant knowledge gap regarding the lives of adults on the autism spectrum. Some literature suggests significant health and mental health inequalities for autistic adults, yet there is a lack of comprehensive Iongitudinal studies exploring risk factors. Further, most research does not include the perspective of autistic adults in its conduct or design. Here, we describe the baseline characteristics and inclusive research approach of a nationwide longitudinal study.

Participants The Autism Cooperative Research Centre for Living with Autism's Australian Longitudinal Study of Adults with Autism (ALSAA) is a questionnaire-based longitudinal study of autistic adults (25+ years old) with follow-up at 2year intervals. Autistic advisors were involved in each stage of research apart from data analysis. Three questionnaires were developed: self-report, informant report (ie, proxy report) and carers (ie, carer experiences and characteristics). Findings to date An inclusive research protocol was developed and agreed with autistic advisors. Baseline data were collected from 295 autistic adults ( $M=41.8$ years, $S D=12.0$ ) including 42 informant responses, 146 comparison participants and 102 carers. The majority of autistic participants $(90 \%)$ had been diagnosed in adulthood $(M=35.3$ years, $S D=15.1)$. When compared with controls, autistic adults scored higher on self-report measures of current depression and anxiety. Participant comments informed ongoing data gathering. Participants commented on questionnaire length, difficulty with literal interpretation of forced response items and expressed gratitude for research in this area.

Future plans A large comprehensive dataset relating to autistic adults and their carers has been gathered, creating a good platform for longitudinal follow-up repeat surveys and collaborative research. Several outputs are in development, with focus on health service barriers and usage, caregivers, impact of diagnosis in adulthood, further scale validations, longitudinal analyses of Ioneliness, suicidal ideation, mental illness risk factors and other areas. Baseline data confirm poorer mental health of autistic adults. The ALSAA demonstrates a working approach to inclusive research.

\section{INTRODUCTION}

Although autism is a lifelong condition, most autism-related research efforts and
Strengths and limitations of this study

- Autism-related research efforts and funding has typically focused on the period from infancy to mid childhood, this research focuses on autistic adults and their carers.

- The Australian Longitudinal Study of Adults with Autism (ALSAA) uses an inclusive research approach to gather a comprehensive range of measures addressing identified research priorities.

- A large proportion of the ALSAA sample at time 1 were female, including $50 \%$ ( $n=149)$ of autistic and $80 \%(n=117)$ of non-autistic participants.

- Participants self-report their autism diagnosis, and a subsample $(n=32)$ is not yet formally diagnosed.

- The ALSAA is a unique resource for exploring multiple research questions regarding the lives of Australian autistic adults.

funding has focused on the period from infancy to mid childhood. ${ }^{1}$ The best available evidence suggests an adult population prevalence of at least $1.1 \% .^{2}$ It is likely that autism is under-recognised or underdiagnosed in adults, ${ }^{3}{ }^{4}$ particularly in women without intellectual disability. ${ }^{25}$ Although an emerging body of research explores outcomes and support needs of autistic individuals beyond childhood and adolescence, studies including autistic adults remain frequently focused on younger adults. ${ }^{6-8}$ This is reflected in recent commentary from an autistic researcher, 'We know virtually nothing about what happens to us, autistic people, physically, cognitively, emotionally and socially as we pass through middle and older age' (Michael, p515). ${ }^{9}$

The growing body of evidence suggests higher rates of physical and mental health conditions, increased mortality and higher rates of intellectual disability than in the general population. ${ }^{10}$ Autistic adults have 
higher prevalence of chronic medical conditions, including conditions such as stroke, vision and hearing impairments, and Parkinson's disease. ${ }^{1-14}$ These outcomes are experienced by many autistic adults regardless of intellectual functioning. ${ }^{7}$ It has been reported that between $25 \%$ and $84 \%$ of autistic adults have a diagnosed mental health condition, with depression and anxiety the most commonly reported conditions. ${ }^{15-19}$ Autistic adults have higher rates of suicide, ${ }^{1620}$ increased mortality and reduced life expectancy. ${ }^{21}$ Despite widespread acknowledgement of the comorbidity between autism and intellectual disability, prevalence estimates of intellectual disability in autism and vice versa have been inconsistent. ${ }^{22}$ Earlier studies report that between $40 \%$ and $70 \%$ of those with autism also have an intellectual disability ${ }^{23-25}$ though a more recent study reports fewer adults on the autism spectrum as having intellectual disability ${ }^{2}$ and intelligence tests may underestimate performance of autistic individuals. ${ }^{26}$

Studies investigating outcomes related to daily activities and participation are also scarce. ${ }^{27}$ Available studies report low employment rates,${ }^{28}$ low community inclusion and limited social relationships for autistic adults. ${ }^{29-33}$ A few studies have suggested that factors such as low childhood IQ, autism severity, psychopathology, social and language development, and access to services and intervention programme may be determinants of such outcomes $^{29}{ }^{34}$; however, much remains to be understood about the sources of variability in these outcomes.

In general, much of the research remains limited by cross-sectional designs, mixed groups with and without intellectual disability, and a focus on adults aged under $40 .{ }^{74-36}$ Longitudinal studies are crucial for exploring changing requirements over time and to inform services how best to respond to the needs of autistic adults. ${ }^{9}{ }^{37}$ While most studies have focused on one aspect of life or outcomes, such as physical or mental health comorbidities $^{121538}$ or quality of life, ${ }^{30}{ }^{39}$ very few comprehensive evaluations of health, well-being and participation have been conducted..$^{34}$ Further, research funding does not match priorities identified by autistic adults, which include research on public services, improving life skills and the future for autistic adults. ${ }^{40}$

The majority of existing autism research is also limited by a lack of inclusive research practices. There is a small but growing body of research on inclusive practices and coproduction in disability research with increasing examples in the autism field. ${ }^{41-43}$ In principle, inclusive research must 'address issues that really matter' to people with disability, 'must access and represent their views and experiences' and people 'need to be treated with respect by the research community' (Walmsley and Johnson, p16). ${ }^{44}$ Inclusive research encompasses a variety of approaches, from including the individuals with the disability as advisors or consultants, to working alongside academic researchers, or initiating or conducting the project as researchers themselves. ${ }^{45}$ Due to a history of mistrust between researchers and autism self-advocacy groups, inclusive methods are especially important in research involving adults on the spectrum. ${ }^{46}$ Inclusive approaches ensure scientific findings are meaningful to the autism community and sensitive to the lived experiences of individuals on the spectrum. ${ }^{47} 48$

The Cooperative Research Centre for Living with Autism (Autism CRC) Australian Longitudinal Study of Adults with Autism (ALSAA) is Australia's first national longitudinal study involving autistic adults aged 25 years and older. The ALSAA will contribute needed longitudinal data across a range of health, functioning and participation domains. Data gathered aligns with the research gaps identified above, and the priorities identified by autistic adults. ${ }^{49}$ In comparison to current international longitudinal studies of autistic adults ${ }^{50-52}$ the ALSAA gathers a wider range of data points using standardised measures and importantly included autistic adults in study design from the early stages of the project. Further, the ALSAA gathers data from a community comparison group, which is often lacking in prior work. The ALSAA will contribute to the validation of several standardised measures for this population. Importantly, the ALSAA gathers data specific to the unique context and service systems within Australia, pertinent given the recent introduction of the National Disability Insurance Scheme and the considerable differences in approaches to healthcare and disability across countries. The ALSAA primarily aims to identify and comprehensively describe the profiles of Australian autistic adults and to compare them to a control group. Longitudinal data will further allow the determination of predictors versus correlates across a range of outcomes. The aims of this paper are to describe the methodological processes and the inclusive protocol adopted in the ALSAA, as well as to present participant characteristics and feedback from the baseline data collection. Exploratory baseline comparisons aim to identify covariates or confounders for consideration in future studies.

\section{COHORT DESCRIPTIONS \\ Design}

The ALSAA is a questionnaire-based, prospective, longitudinal cohort study with a planned 2-year follow-up and further follow-ups as resources allow. Initial design and development of the ALSAA occurred in collaboration with researchers and clinical health professionals across Australia, including close collaboration with the Autism CRC longitudinal Study of Australian School Leavers with Autism (SASLA) ${ }^{53}$ Brief consultation also occurred with international experts and researchers conducting similar longitudinal studies. Additionally, preliminary work on factors influencing the research participation of autistic adults ${ }^{54}$ influenced the ALSAA design, including ensuring opportunities for participants to clarify questions, receive 
regular study newsletters and space for participants to provide explanations regarding their responses.

\section{Patient and public involvement}

How was the development of the research question and outcome measures informed by patients' priorities, experience and preferences?

The ALSAA was developed in conjunction with SASLA, the SASLA team conducted a stakeholders' research forum in late 2014 and obtained written feedback from participants (autistic people, families and service providers) that helped inform both studies. The SASLA team also consulted with individuals on the spectrum, parents and autism support groups. The ALSAA was developed with input from a research advisory network, initially consisting of six autistic adults and two family members/carers of autistic adults who helped determine areas of investigation, and develop the surveys to ensure the questions were relevant, accessible and valid.

\section{How did you involve patients in the design of this study?}

The ALSAA Inclusive Research Protocol was developed from interactions between the researchers and advisors, and based on the principles of inclusive research, ${ }^{55}$ the Autism CRG report Inclusive Research Practice Guides and Checklists for Autism Research ${ }^{56}$ (eg, checklist 3: Practices that Support Inclusion of Individuals on the Autism Spectrum in Advisory and Reference Groups), and procedures similar to those employed by Nicolaidis et $a l^{p 7}$ (eg, choosing topic areas, adapting data collection instruments, interpreting results) (see table 1). In contrast to Nicolaidis et $a l,{ }^{57}$ ALSAA employs an advisory approach as opposed to participatory approaches where final decision-making rests with the committee, and there has not been any group or in-person consultation sessions, which is a preference of some autistic advisors. Guidelines such as those developed by AASPIRE $^{58}$ were not available at the time of the study design.

\section{Were patients involved in the recruitment to and conduct of the study?}

Since the commencement of the ALSAA, this inclusive research protocol has been reviewed by seven of the autistic advisors and one carer advisor and has guided the interactions between researchers and advisors in the development of baseline outputs and the 2-year follow-up design. Specifically, in the design stage of the ALSAA, members of the advisory network were asked to identify ambiguous or inappropriate language, formatting issues and measures which they thought may be interpreted inappropriately or differently due to poor clarity or wording. An expanded group of eleven advisors had greater input in designing the 2-year follow-up, including the selection of focus topic areas. A list of topic areas was suggested to the advisors based on a review of time 1 participant feedback. Advisors reviewed the list and added more topics or specific research questions of interest. A few advisors expressed difficulty or did not complete a task of ranking the topics, hence the researchers were required to make the final shortlisting of new topics areas to be included in data gathering. In comparison to several other studies, ${ }^{59}$ autistic adults are involved in the majority of stages of the study apart from collecting data, they have been involved in review of some media related lay summaries but have not typically been involved in development of newsletter content. As per the protocol advisors are given forewarning, then 1 month to respond to most requests from the research team, with more response time as needed. Advisors have provided feedback on ongoing research outputs critiquing interpretations of findings suggested by the researchers. In time 2 data gathering and analyses, compensation has been made available for advisors' time, on request to the Autism CRC, which has become increasingly supportive of co-production approaches. Advisors are now offered an hourly rate for each time they are consulted, with most consultations limited to 2 hours, though several advisors still prefer to volunteer and do not request compensation on most occasions. Advisors to date have not been involved in data analysis, though several coproduced peer-research projects are currently underway. Advisors have also assisted in promoting recruitment materials particularly through social media.

\section{How will the results be disseminated to study participants?}

The ALSAA sends quarterly digital newsletters to all participants. Newsletters include lay summaries of any publications, conference presentations or other outputs developed using the ALSAA data. They also typically contain general interest highlight(s) relating to the autism or autistic community, recruitment calls for other autism research, as well as a team member profile of an ALSAA researcher or autistic advisor. Advisors frequently assist in furthering the promotion of the study and outputs via social media. Our recent study on loneliness, ${ }^{60}$ included in the latest study newsletter, was a topic originally identified by advisors in time 1 data gathering, and demonstrates the inclusive approach used in the ALSAA. Advisors have been approached to assist with autism-related media enquiries and will continue to do so when opportunities arise.

\section{Participants}

There are three participant groups in the ALSAA: autistic adults, non-autistic general community comparison controls and carers of autistic adults. Inclusion criteria required the control and autistic participants to be aged 25 years or older, live in Australia and have sufficient English literacy skills to complete the survey, determined during a screening phone call or online expression of interest (EOI). If an autistic individual was unable or preferred not to self-report, they were given the option to ask an informant to complete an informant version of the survey on their behalf. People with intellectual disability could complete the self-report survey with support or participate via an informant respondent.

Informant respondents and carers needed to be 18 years or older, have sufficient English literacy skills, live in Australia 
Table 1 The ALSAA inclusive research protocol

\begin{tabular}{ll}
\hline Step & Description of step and considerations \\
\hline $\begin{array}{l}\text { Step 1: recruitment of } \\
\text { advisors/peer-researchers }\end{array}$ & $\begin{array}{l}\text { Ensure recruitment is inclusive of all potential autistic individuals including adults with intellectual disability } \\
\text { consider using multiple channels of recruitment (eg, Autism associations, self-advocacy networks, carer } \\
\text { networks, adult networks, local radio, social media and word of mouth). In Australia, seek support from the }\end{array}$ \\
& $\begin{array}{l}\text { Autism CRC Research Academy. } \dagger \\
\text { Step 2: building rapport }\end{array}$ \\
$\begin{array}{l}\text { If an established relationship does not already exist between the autistic individual and researcher, the researcher } \\
\text { should offer to meet the individual (either face to face, over video conference or phone call). Be mindful that } \\
\text { some autistic individuals may prefer to communicate via text or email and not to meet in person and should not } \\
\text { be required to. }\end{array}$
\end{tabular}

Step 3: clarify what will be expected from the advisor

Outline tasks autistic advisors or peer researchers will be asked to complete. Specify the frequency and timing of when they are likely to be contacted. For specific tasks clearly provide step-by-step instructions, flexible leadtime, timeframes for feedback, area of investigation and broad aim of the study. Make information available in Easy English or plain language as appropriate. Specify the type and nature of advice sought. Clearly describe how and where their input will be used and why their input is being sought.

Step 4: ethics, confidentiality Describe ethics of the project and expected confidentiality. As required, specify on individual documents that and acknowledgement the document is confidential and not to be distributed without discussion with the researcher. Can the autistic individual opt out if they would like? Outline what will happen if they choose to withdraw, for example, this will not affect their relationship with the research team, university or any other associated organisations. It is a voluntary process.

Negotiate and make explicit how the autistic advisor or peer-researcher will be acknowledged. Consider how the autistic individual may be recognised in a manuscript or research output. This may include as a coauthor, in the acknowledgements section and/or in discussing methodology and findings.

$\begin{array}{ll}\text { Step 5: identify preferred } & \text { Ask the autistic individual their preferred communication style for providing feedback (eg, face to face, phone } \\ \text { communication style } & \text { call, email, video or a variety of techniques). Also, in what medium they would like to receive the results/findings, } \\ \text { for example, academic writing style, easy English writing style, mostly graphs/pictures, mostly dot points, } \\ \text { described in person, video, PowerPoint presentation. Although not all mediums may be practical, it is important } \\ \text { to identify the autistic advisor's preferred style and then work together to identify a medium of communication } \\ \text { which will be understood and accessible by both the autistic advisor and the researcher. }\end{array}$

Step 6: the sensory environment

Step 7: involvement in methodological design/ results/findings
The researcher must provide an 'autism-friendly' environment on any occasion where the autistic advisor and the researcher may be meeting in person, see p. 12-15 in the Autism CRC Inclusive Research Practice Guides and Checklists for Autism Research ${ }^{56}$ regarding creating autism-friendly environments.

Autistic advisors input is sought in regard to areas of investigation, questionnaire design and interpretation of results. The researcher will need to consider that ranking of priority areas may be a difficult task for some advisors and employ other consensus decision-making strategies. The researcher will need to present questionnaire design questions or quantitative or qualitative results in an understandable format, developing design issue summaries or results summaries in the medium preferred by the autistic advisor or peer-researcher. This means the researcher may need to make a number of decisions around data analysis and interpretation. Where possible, outline each of these decisions so the process is transparent and the autistic advisor can reflect on the decisions made by the researcher and whether these were appropriate in their view. Provide clear guidance on what advice is being sought, provide lead time, and the time frame and process to follow for providing advice. How long should the advice be? In what format? Who will be reading it, who do they send the advice to? What are the researchers specifically looking for, what should the advice focus on?

Step 8: feedback

Once the researcher receives the advice from the autistic advisor, the researcher should provide a time frame in which the autistic advisor will receive an update on what aspects of the advice was incorporated and the effect it had/did not have on the methodology, findings and/or interpretation.

Step 9: final checks

Once the manuscript or research output has been further developed, the autistic advisor should receive a copy of the manuscript prior to submission. If the autistic advisor has limited experience with academic writing, the researcher should offer to describe/provide a lay summary of the overall article for the advisor.

Step 10: acknowledgement Autistic advisor or peer-researcher to be acknowledged in the agreed on manner (eg, coauthor, thanked in the acknowledgement section, acknowledged during a presentation, etc).

*Although inclusive consultation occurred at time 1, the ALSAA RAN does not currently have an active member on the autism spectrum with an intellectual disability. Renewed efforts to recruit an advisor will be instigated when autism and intellectual disability focused outputs are commenced. We consult as needed with an advisor with intellectual disability not on the autism spectrum currently.

†The Autism CRC Research Academy (https://www.autismcrc.com.au/research-academy) provides training for peer researchers.

ALSAA, Australian Longitudinal Study of Adults with Autism; CRC, Cooperative Research Centre for Living with Autism.

and be able to report on the life of the autistic person. The content of the informant survey is slightly different to the self-report survey (see table 2) due to some measures, such as the Patient Health Questionnaire-9 (PHQ-9), ${ }^{61}$ having no informant version available. Carers, including family members and support people for the ALSAA, were defined as people who provided unpaid care and support to either a family member (including spouse) or friend who is on the autism spectrum. This is based on the widely accepted definition proposed by Carers Australia (http://www.carersaustralia.com.au/about-carers/). 
Table 2 Measurements included in the ALSAA surveys

\section{Measure/domain}

\section{Self-report Informant report Carer}

Demographics and personal characteristics

Basic demographics

(age, sex, ethnicity, English speaking status)

Other demographics

(education, occupation*, family and living conditions, income)

Autism characteristics

\begin{tabular}{|c|c|c|c|}
\hline The Autism Quotient-Short† (AQ-Short) ${ }^{67}$ & $\checkmark$ & $\checkmark$ & $\checkmark$ \\
\hline Repetitive Behaviours Questionnaire-2 adult version ${ }^{63}$ & $\checkmark$ & $\checkmark$ & \\
\hline The Glasgow Sensory Questionnaire ${ }^{95}$ & $\checkmark$ & $\checkmark$ & \\
\hline The Intolerance of Uncertainty Scale- $12^{96}$ & $\checkmark$ & & $\checkmark$ \\
\hline \multicolumn{4}{|l|}{ lealth and well-Being } \\
\hline Medical history & $\checkmark$ & $\checkmark$ & $\checkmark$ \\
\hline Medication profile & $\checkmark$ & $\checkmark$ & \\
\hline Smoking, alcohol and illicit drug use* & $\checkmark$ & $\checkmark$ & \\
\hline The Patient Health Questionnaire-15 (PHQ-15) ${ }^{97}$ & $\checkmark$ & & $\checkmark$ \\
\hline Medical outcomes study Short Form Health Survey-12 ${ }^{98}$ & $\checkmark$ & $\checkmark$ & $\checkmark$ \\
\hline The Composite Autonomic Symptom Score-31 ${ }^{99}$ & $\checkmark$ & & \\
\hline Pittsburgh Sleep Quality Index ${ }^{100}$ & $\checkmark$ & & \\
\hline Flinders Fatigue Scale $e^{101}$ & $\checkmark$ & & \\
\hline
\end{tabular}

Mental and emotional health

DSM-5 Dimensional Anxiety Scales: Generalised Anxiety Disorder-Adult $(\mathrm{DSM}-5)^{102}$

The PHQ-961

The Warwick Edinburgh Mental Well-being Scale ${ }^{103}$

The New General Self-efficacy Scale ${ }^{104}$

The Emotion Regulation Questionnaire ${ }^{105}$

Developmental Behaviour Checklist-Adolescent/Adult version Revised ${ }^{106}$

The Brief COPE ${ }^{107}$

Memory Complaint Questionnaire ${ }^{108}$

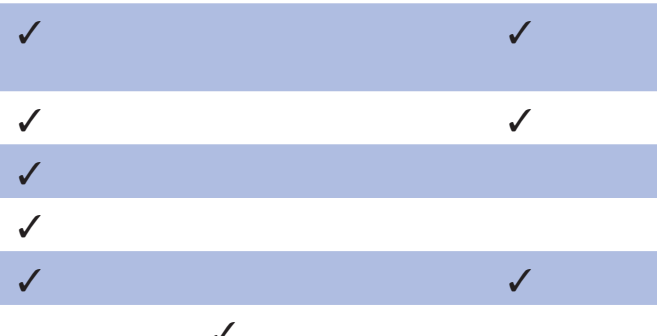

Relationships and social networks

UCLA Loneliness Scale-8 ${ }^{109}$

Supports and networks

The Social Support Questionnaire ${ }^{110}$

Activities, participation and quality of life

World Health Organisation Quality of Life (WHOQOL) -BREF ${ }^{111}$

Waisman Activities of Daily Living Scale ${ }^{112}$

WHO Disability Assessment Schedule ${ }^{113}$

Vocational index for adults with autism ${ }^{114}$

Special abilities and interestsł

Leisure activities participation

Leisure Satisfaction Scale ${ }^{115}$

Driving and transport

Service usage

Continued 
Table 2 Continued

\begin{tabular}{lcc}
\hline Measure/domain & Self-report & Informant report \\
\hline Client Service Receipt Inventory ${ }^{116}$ & $\checkmark$ & $\checkmark$ \\
Health service seeking behaviour & $\checkmark$ & $\checkmark$ \\
Early intervention service history & $\checkmark$ & $\checkmark$ \\
Justice system contact & $\checkmark$ & $\checkmark$ \\
Preventative health screening & $\checkmark$ & $\checkmark$
\end{tabular}

Caring

Positive Affect Index ${ }^{117}$

Caregiver Activity Survey-Intellectual Disability ${ }^{118}$

Zarit Burden Interview ${ }^{119}$

*Based on questions from the National Health Survey of Mental Health and Well-being (Slade, Johnston, Oakley Browne, Andrews and Whiteford, 2009).

†A 28-item parent-carer report adaptation using items from the AQ-Adolescent (Baron-Cohen, Hoekstra, Knickmeyer and Wheelwright, 2006) was used for the Informant-Report Survey.

$\ddagger$ Based on questions from the Interactive Autism Network (https://iancommunity.org/).

ALSAA, Australian Longitudinal Study of Adults with Autism.

\section{Survey}

Three versions of the survey were developed: (1) selfreport, for autistic adults and non-autistic controls, (2) informant, for carers/support people to complete about the autistic person who is unable to, or does not want to self-complete and (3) carer survey for carers/support people/family members to complete about their own health and well-being. Surveys could be completed via a paper copy or online. The paper copy was also available in large print. The surveys involved a comprehensive range of measures (see table 2). In selecting measures, preference was given to measures that had been previously used or had established validity with people on the spectrum and to those deemed suitable by the autistic advisors. Most measures have not been specifically validated for autistic adults, with notable recent exceptions of the WHOQOL BREF, ${ }^{51}$ Short Form Health Survey- $12^{62}$ and the Repetitive Behaviours Questionnaire-2 Adult Version, ${ }^{63}$ while the PHQ-9 ${ }^{64}$ was more recently validated using a combined ALSAA and SASLA data set.

Each survey also contained closing feedback items, asking if any questions were confusing, difficult or upsetting, and any additional topics that should be included. It also asked how long the survey took to complete (openended response), and an overall satisfaction with the survey rated on a 5-point Likert scale. A final item asked for any further feedback.

\section{Procedure}

Piloting

Four autistic adults piloted the self-report questionnaire and two caregivers piloted the carer and the informant questionnaires. Volunteers received two copies, one copy to complete, with data recorded where possible in the ALSAA dataset, and the other for their feedback. Considerable changes to each version of the survey were undertaken in response to feedback from the autistic advisors and volunteers, including removing the Communication Checklist-Adult, ${ }^{65}$ formatting changes and changes to item wordings where the item did not belong to an existing standardised tool.

\section{Recruitment and follow-up}

A recruitment target of 160 autistic adults was set to allow for regression analyses using multiple predictor variables. Power analyses, undertaken using power calculation software, suggested this would be a sufficient sample size with power $(1-\beta)$ set at 0.80 and $\alpha=0.05$ to detect a Cohen's $f^{2}$ effect size estimate of at least 0.1 using multiple regression models with seven predictors. Participants were recruited via advertisements through autism-specific organisations, disability organisations, autism self-advocacy groups, employment services, universities, Technical and Further Education institutes, psychology and allied health private practices, carer organisations and online autism communities. In attempts to gather a sample more closely matched to Australian population estimates, ${ }^{66}$ additional recruitment activities targeted adults on the spectrum with intellectual disability and males without autism. General community members, autistic adults and carers of autistic adults who were willing to participate in the ALSAA contacted the research team based at The University of New South Wales (UNSW Sydney) via telephone, email or through completing a short online EOI. After eligibility screening, their details were added to the participant database and they were mailed a paper copy or emailed a link to their survey depending on their preference.

Due to a high number of responses and advice from autistic advisors, participants who self-identified as being autistic, but did not have a formal diagnosis, representing the hidden population and 'lost generation' ${ }^{3}$ of autistic 
adults, were also recruited. Formally diagnosed participants were asked to provide details of their diagnosis, year of diagnosis, and the discipline and name of the practitioner(s) who diagnosed them. Autism Quotient-Short (AQ-short) cut-off scores ( $>65$, sensitivity 0.97 , specificity $0.82)^{67}$ also support diagnostic status. Given critiques of applicability and validity of the AQ-short, ${ }^{6869}$ we plan to include the Ritvo Autism and Asperger Diagnostic Scale$14^{69}$ in future data gathering. At the planned 2-year average time 2 follow-up copies of participants' diagnostic reports will be requested, with a project undertaken to review and categorise the reports. From November 2017 to 2019, participants are being recontacted using contact details provided and considering their communication preferences that were recorded.

\section{Ethical considerations}

Participants were supplied with the appropriate participant information statement and consent form (PISCF). For the online survey, participants were asked to confirm their consent at commencement, marking checkboxes prior to proceeding. Consent was inferred from participants who completed and returned paper copies of the survey, as outlined in their PISCF. In the case where the autistic adult had an intellectual disability a checklist for capacity to provide consent was used. This checklist was completed by the carer/next of kin; it was adapted from a checklist developed for use in the Study of Ageing in Intellectual Disability. ${ }^{70}$ The checklist asks several question relating to the person's ability to understand the study, benefits and risks, withdrawal and complaints, to determine if a person responsible consent should be obtained additionally or separately.

\section{Data storage}

Online questionnaire data were entered directly by participants into Qualtrics Online Survey Software (https://www.qualtrics.com/), whereas returned paper copy surveys were entered into Qualtrics by a researcher or research assistant. Data from Qualtrics, reidentifiable by participation code, were stored on password-protected UNSW Sydney servers. Recently, ethics approval for establishment of a databank for the ALSAA data, to allow collaboration with external researchers, has been obtained.

\section{Statistical analysis}

STATA Statistical Analysis Software V.14 ${ }^{71}$ and IBM SPSS Statistics V.22 $2^{72}$ were used for cleaning, coding and statistical analyses. For the current manuscript, STATA was used to obtain descriptive statistics regarding recruitment, participant feedback and participant characteristics, with the level of significance $<0.05$. No correction was applied for multiple statistical tests in this manuscript, as the purpose was to explore baseline differences in the cohorts, and determine potential variables that may be useful covariates or possible confounders in later hypothesis testing. ${ }^{73}$ For describing this cohort profile, listwise deletion was used for specific analyses when data were missing completely at random, after checking for difference in demographics and AQ score for dropped participants where appropriate. Subsample sizes are noted throughout. Data were checked for kurtosis and skew. Non-parametric Spearman rank correlation was used to determine the correlation between time taken to complete the self-report survey and level of satisfaction.

\section{Recruitment statistics}

Recruitment commenced in July 2015. At the close of time 1 data gathering in November 2017, 345 autistic adults, 221 non-autistic adults, 185 carers and 78 informants had registered their interest in the study. Of these, 237 autistic adults, 130 non-autistic adults, 100 carers and 42 informant reporters completed the entire survey. Overall, a total of 295 autistic adults participated including all survey formats and partial survey completions. A small proportion of participants were deemed ineligible during screening (see table 3), generally due to being under 25 years, not caring for an adult aged 25 +yearsold, or being located outside of Australia. Participants were recorded as partial completions if at close of time 1 their surveys contained the initial demographic items completed and some additional useable data. From available data, there was no significant difference in binary gender, $\mathrm{X}^{2}(4, \mathrm{n}=337)=1.07, \mathrm{p}=0.898$ or approximate age $(\mathrm{F}(4,4)=2.04, \mathrm{p}=0.09)$ based on survey commencement, eligibility or completion status for the self-reporting autistic adults (see table 1). These nonsignificant results were also found for the non-autistic adults across gender, $\mathrm{X}^{2}(4, \mathrm{n}=215)=6.98, \mathrm{p}=0.137$ and age $(\mathrm{F}(4,4)=0.96, \mathrm{p}=0.43)$.

Table 3 Recruitment statistics at close of time 1 data gathering

\begin{tabular}{|c|c|c|c|c|c|c|}
\hline Sample & Registered & Non-respondent & $\begin{array}{l}\text { Ineligible } \\
(\%)\end{array}$ & $\begin{array}{l}\text { Withdrawn } \\
(\%)\end{array}$ & $\begin{array}{l}\text { Partial } \\
\text { completion (\%) }\end{array}$ & $\begin{array}{l}\text { Completed } \\
(\%)\end{array}$ \\
\hline Self-report autistic adults & 345 & $75(22)$ & $6(2)$ & $11(3)$ & $16(5)$ & $237(69)$ \\
\hline Carer & 185 & $48(26)$ & $22(12)$ & $13(7)$ & $2(1)$ & $100(54)$ \\
\hline $\begin{array}{l}\text { Informant-report for autistic } \\
\text { adult }\end{array}$ & 78 & $22(28)$ & $5(6)$ & $9(11)$ & $0(0)$ & $42(54)$ \\
\hline
\end{tabular}




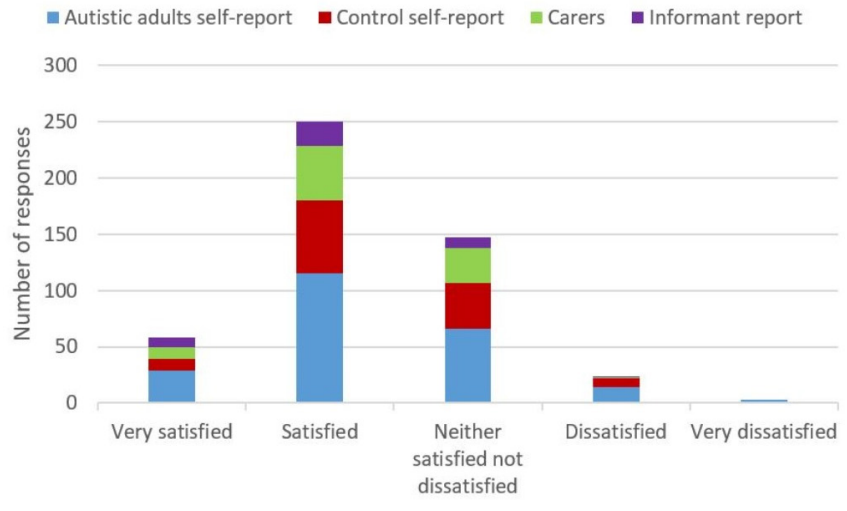

Figure 1 Satisfaction responses on completion combined by survey type $(n=482)$.

\section{Time taken to complete, and satisfaction with, the survey}

Participants reported varying times taken to complete the entire survey. Self-reporting autistic participants reported between $20 \mathrm{~min}$ and 12 hours to complete with an average time of $156 \mathrm{~min}(\mathrm{n}=164, \mathrm{SD}=111)$, though several responses $(n=53)$ were not easily convertible to numeric values for analysis, for example 'few days', 'no idea' or no response to the item $(n=36)$. Non-autistic, self-reporting participants reported a range of $20 \mathrm{~min}$ to 5 hours with an average time of $106 \mathrm{~min}(\mathrm{n}=98, \mathrm{SD}=74)$. This was significantly less than self-reporting autistic adults despite their completing almost all the same measures and domains, $\mathrm{t}(260)=-3.94, \mathrm{p}<0.001, \mathrm{~d}=-0.50$. Carers took $75 \mathrm{~min}$ on average $(\mathrm{n}=81, \mathrm{SD}=62)$ and informant responders took $103 \mathrm{~min}$ on average $(\mathrm{n}=27, \mathrm{SD}=54)$ to complete surveys. Most participants $(64 \%, \mathrm{n}=482)$ reported that they were satisfied or very satisfied with the survey; few participants were dissatisfied (6\%) (figure 1). There was no association between time taken to complete the self-report survey and level of satisfaction $\left(n=262, r_{s}=0.10, p=0.09\right)$, and no difference in satisfaction based on survey type, $\mathrm{X}^{2}(9, \mathrm{n}=482)=11.2, \mathrm{p}=0.26$.

Across self-reporting autistic adults, the most common negative feedback related to difficulty with literal interpretations of forced response items $(n=38)$ where participants felt the options provided did not match their circumstances, or to the length of the survey $(n=17)$. Several participants provided feedback that they were grateful for research in this area $(n=27)$. Many additional topics of interest were suggested, with the most common being relationships, bullying, gender and sexuality, and a more detailed employment section. The potential removal of some questionnaires (eg, the Brief COPE) was also commented on. Revision of items referring to social supports was suggested, as some participants found these upsetting $(n=12)$ and/or confusing $(n=7)$, with comments such as 'I had to really think about my social circle and realised the limited number of truly reliable and trustworthy friends I actually have, which was kind of depressing'.

\section{Summary of participant characteristics}

The age of autistic participants ranged from 25 to 80 years, with a high proportion of females $(50 \%, \mathrm{n}=295)$ across all autistic samples. Across all autistic participants, 36\% were living as a couple with $25 \%$ of the sample currently married. Over half $(57 \%, \mathrm{n}=42)$ of the informant report sample lived with their parents (see table 4), in contrast to self-reporting autistic adults who most frequently lived as a couple $(45 \%, \mathrm{n}=295)$ or alone $(26 \%, \mathrm{n}=295)$. Self-reporting autistic adults did not differ from control participants on rural/remoteness according to Australian Statistical Geography Standard-Remoteness Area classification, $\mathrm{X}^{2}(4, \mathrm{n}=378)=9.36, \mathrm{p}=0.05, \mathrm{~V}=0.16(\mathrm{n}=21 \mathrm{missing})$, with no control participants and minimal autistic participants living in remote or very remote Australia. Propensity to greater socioeconomic disadvantage according to the postcode-based Socioeconomic Index for Areas (SEIFA) decile was significant, $\mathrm{t}(376)=2.96, \mathrm{p}<0.01, \mathrm{~d}=0.32(\mathrm{n}=21$ missing, NB. SEIFA/rurality not available for all postcodes), though they were still above average compared with the Australian population. Time 2 data gathering will seek to gather household income data to more accurately determine socioeconomic disadvantage.

Of the formally diagnosed autistic adults ( $n=221), 132$ (56\%) reported they have a diagnostic report available, with $169(76 \%)$ diagnosed by one clinician and $29(13 \%)$ reporting diagnosis by two or more clinicians $(n=23$ missing). As shown in table 5, the majority $(97 \%, \mathrm{n}=295)$ of all autistic participants met the AQ-Short cut-off for identifying autism, compared with $30 \%(n=60)$ of biologically related carers and 22\% $(n=137)$ of non-autistic control participants. Asperger's syndrome was the most frequently reported formal diagnosis. Self-reporting, formally diagnosed autistic participants on average were diagnosed 6.9 years prior to survey completion, and the majority $(90 \% ; \mathrm{n}=217,4$ missing) received their diagnosis in adulthood. A preliminary analysis of gender differences for self-reporting autistic adults was also conducted. In comparison to males, self-reporting formally diagnosed females did not differ significantly on age of diagnosis $\mathrm{t}(192)=.39, \mathrm{p}=0.70, \mathrm{~d}=0.06 \quad(\mathrm{n}=4$ missing $)$. Comparing males with females, including non-diagnosed autistic adults, there was no difference in number of individuals currently married or in a de facto relationship, $\mathrm{X}^{2}(1$, $\mathrm{n}=241)=0.78, \mathrm{p}=0.38, \mathrm{~V}=-0.06$, or in their educational status, $\mathrm{X}^{2}(2, \mathrm{n}=241)=4.66, \mathrm{p}=0.10, \mathrm{~V}=0.14$. Females were significantly different regarding employment status $\mathrm{X}^{2}(4$, $\mathrm{n}=219)=18.77, \mathrm{p}=0.001, \mathrm{~V}=0.29$, though only relating to the number providing homecare $(n=19$ female vs $\mathrm{n}=1$ male, see table 4 ).

In the non-autistic control sample, participant ages ranged from 25 to 79 years, with a high proportion $(80 \%, n=146)$ of females. The majority lived as a couple $(72 \%)$ and were married $(62 \%)$. The control sample was significantly different from the self-reporting autistic adults (with and without a formal diagnosis) on several demographics including gender, $X^{2}(2, n=399)=26.5$, $\mathrm{p}<0.001, \mathrm{~V}=0.26$, living situation, $\mathrm{X}^{2}(5, \mathrm{n}=399)=33.2$, 
Table 4 Participant characteristics

\begin{tabular}{|c|c|c|c|c|c|}
\hline Characteristic & $\begin{array}{l}\text { Autistic adults } \\
\text { self-report formally } \\
\text { diagnosed ( } n=221)\end{array}$ & $\begin{array}{l}\text { Autistic adults self- } \\
\text { report no formal } \\
\text { diagnosis }(n=32)\end{array}$ & $\begin{array}{l}\text { Autistic adults } \\
\text { informant-report } \\
(n=42)\end{array}$ & $\begin{array}{l}\text { Carers } \\
(\mathrm{n}=102)\end{array}$ & $\begin{array}{l}\text { Non-autistic } \\
\text { self-report } \\
(n=146)\end{array}$ \\
\hline Age mean (SD) & $41.8(12.2)$ & $44.2(8.1)$ & $32.5(9.9)$ & $56.3(11.2)$ & $43.7(13.5)$ \\
\hline \multicolumn{6}{|l|}{ Gender (\%) } \\
\hline Male & $86(39)$ & $14(44)$ & $34(81)$ & $16(16)$ & $29(20)$ \\
\hline Female & $124(56)$ & $17(53)$ & $8(19)$ & $85(83)$ & $117(80)$ \\
\hline Other & $11(5)$ & $1(3)$ & 0 & $1(1)$ & 0 \\
\hline \multicolumn{6}{|l|}{${ }^{*}$ Ethnicity (\%) } \\
\hline Caucasian & $194(88)$ & $25(78)$ & $38(90)$ & $86(89)$ & $122(85)$ \\
\hline Other & 27 (12) & 7 (22) & $4(9)$ & $11(11)$ & $22(15)$ \\
\hline Multilingual (\%) & $26(12)$ & $5(16)$ & 0 & $6(6)$ & $18(12)$ \\
\hline †SEIFA decile (SD) & $6.18(2.89)$ & $5.74(3.09)$ & $6.58(2.88)$ & $6.60(2.78)$ & $7.01(2.60)$ \\
\hline \multicolumn{6}{|l|}{ †‡Rural/remoteness } \\
\hline Major city & $146(71)$ & $17(55)$ & $32(84)$ & $72(73)$ & $114(83)$ \\
\hline Inner regional & $44(21)$ & $10(32)$ & $5(13)$ & $21(21)$ & $19(14)$ \\
\hline Outer regional & $12(6)$ & $4(13)$ & $1(3)$ & $5(5)$ & $5(4)$ \\
\hline Remote/very remote & $4(2)$ & 0 & 0 & 0 & 0 \\
\hline \multicolumn{6}{|l|}{ §Living situation (\%) } \\
\hline Living alone & $58(26)$ & $9(28)$ & $3(7)$ & 14 (14) & $26(18)$ \\
\hline Living as couple & $95(43)$ & $18(56)$ & $4(9)$ & $74(75)$ & $105(72)$ \\
\hline Living with parents & $31(14)$ & 0 & $24(57)$ & 0 & $5(3)$ \\
\hline $\begin{array}{l}\text { Living with other } \\
\text { relatives }\end{array}$ & $3(1)$ & 0 & $1(2)$ & $6(6)$ & $3(2)$ \\
\hline Living with others & 20 (9) & $5(16)$ & 8 (19) & $4(4)$ & $5(3)$ \\
\hline Other & $14(6)$ & 0 & $2(5)$ & $1(1)$ & $2(1)$ \\
\hline \multicolumn{6}{|l|}{ IMarital status (\%) } \\
\hline Single & $73(33)$ & 7 (22) & $36(86)$ & $4(4)$ & $22(15)$ \\
\hline Married & $66(30)$ & $12(37)$ & $4(9)$ & $65(66)$ & $90(62)$ \\
\hline De facto & $17(8)$ & $3(9)$ & 0 & $8(8)$ & $14(9)$ \\
\hline Never married & $8(4)$ & $3(9)$ & $2(5)$ & $2(2)$ & $3(2)$ \\
\hline Widowed & $1(0)$ & 0 & 0 & $3(3)$ & $2(1)$ \\
\hline Divorced now single & $21(9)$ & $3(9)$ & 0 & $8(8)$ & $9(6)$ \\
\hline $\begin{array}{l}\text { Divorced now } \\
\text { remarried/de facto }\end{array}$ & $12(5)$ & $2(6)$ & 0 & $2(2)$ & $2(1)$ \\
\hline Separated & $7(3)$ & $1(3)$ & 0 & $2(2)$ & $1(1)$ \\
\hline Other & $16(7)$ & $1(3)$ & 0 & $4(4)$ & $3(2)$ \\
\hline \multicolumn{6}{|l|}{ Educational status (\%) } \\
\hline $\begin{array}{l}\text { Completed high } \\
\text { school }\end{array}$ & $163(76) n=215$ & $20(64) n=31$ & $21(51) n=41$ & $86(88) n=98$ & $120(86) n=139$ \\
\hline Vocational education & $61(35) n=174$ & $16(57) n=28$ & $4(11) n=35$ & $17(17) n=98$ & $20(16) n=127$ \\
\hline University education & $113(65) n=174$ & $12(43) n=28$ & $1(3) n=35$ & $59(60) n=98$ & $107(84) n=127$ \\
\hline \multicolumn{6}{|l|}{${ }^{* \star}$ Employment (\%) } \\
\hline Open employment & $116(57)$ & $20(69)$ & $6(15)$ & $59(60)$ & $104(76)$ \\
\hline $\begin{array}{l}\text { Student/other day } \\
\text { activity }\end{array}$ & 38 (19) & $3(10)$ & $24(60)$ & $3(3)$ & 17 (12) \\
\hline $\begin{array}{l}\text { Retired, no } \\
\text { volunteering }\end{array}$ & $8(4)$ & $2(7)$ & $2(5)$ & $19(19)$ & $6(4)$ \\
\hline
\end{tabular}


Table 4 Continued

\begin{tabular}{clllll}
\hline & $\begin{array}{l}\text { Autistic adults } \\
\text { self-report formally } \\
\text { diagnosed }(\mathbf{n}=\mathbf{2 2 1})\end{array}$ & $\begin{array}{l}\text { Autistic adults self- Autistic adults } \\
\text { report no formal } \\
\text { diagnosis } \mathbf{( n = 3 2 )}\end{array}$ & $\begin{array}{l}\text { Aunformant-report } \\
\text { in=42) }\end{array}$ & $\begin{array}{l}\text { Carers } \\
(\mathbf{n}=102)\end{array}$ & $\begin{array}{l}\text { Non-autistic } \\
\text { self-report } \\
(\mathbf{n}=146)\end{array}$ \\
\hline Homecare & $17(8)$ & $3(10)$ & 0 & $9(9)$ & $9(6)$ \\
No activity & $22(11)$ & $1(3)$ & $8(20)$ & $9(9)$ & $1(1)$ \\
\hline
\end{tabular}

${ }^{*}$ Ethnicity: carers, $n=97$; non-autistic (self-report), $n=144$.

†Autistic adults (diagnosis, self-report), $n=209$; autistic adults (no diagnosis, self-report), $n=31$; autistic adults (informant report), $n=38$; carers, $\mathrm{n}=99$; non-autistic (self-report), $\mathrm{n}=138$.

$\ddagger$ Australian population is $70 \%$ major city, $18 \%$ inner regional, $9 \%$ outer regional, $1 \%$ remote, $1 \%$ very remote in the 2011 Census. ${ }^{120}$ $\S$ Carers' living situation: $n=99$.

ๆCarers' marital status: $\mathrm{n}=98$.

**Employment: autistic adults (diagnosis, self-report), $\mathrm{n}=201$; autistic adults (no diagnosis,self-report), $\mathrm{n}=29$; autistic adults (informant report), $n=40$; carers, $n=99$; non-autistic (selfreport), $n=137$.

SEIFA, Socioeconomic Index for Areas.

$\mathrm{p}<0.001, \quad \mathrm{~V}=0.29$, marital status, $\mathrm{X}^{2}(8, \mathrm{n}=398)=47.1$, $\mathrm{p}<0.001, \mathrm{~V}=0.34,(\mathrm{n}=1$ missing $)$, employment status, $\mathrm{X}^{2}(6$, $\mathrm{n}=367)=18.3, \mathrm{p}=0.005, \mathrm{~V}=0.22(\mathrm{n}=32$ missing $)$ and higher education status, $\mathrm{X}^{2}(2, \mathrm{n}=399)=22.4, \mathrm{p}<0.001, \mathrm{~V}=0.24$. Of interest, differences in education and employment were not significant if analyses were limited to male participants. The control sample was also significantly less likely to meet the DSM-5-TR linked PHQ-9 cut-off for depression, $\mathrm{X}^{2}(1, \mathrm{n}=354)=54.9, \mathrm{p}<0.001, \mathrm{~V}=0.39$, ( $\mathrm{n}=45$ missing $)$, less likely meet the DSM-5 Generalised Anxiety DisorderAdult (GAD-A) cut-off ${ }^{74}$ for anxiety, $\mathrm{X}^{2}(1, \mathrm{n}=366)=67.8$, $\mathrm{p}<0.001, \mathrm{~V}=0.43$ ( $\mathrm{n}=33$ missing) and less likely to have a history of psychiatric illness, $\mathrm{X}^{2}(1, \mathrm{n}=384)=82.1, \mathrm{p}<0.001$, $\mathrm{V}=0.46$ ( $\mathrm{n}=15$ missing), compared with self-reporting autistic adults, and remained significant if limited to female or male participants. There were no significant differences for self-reporting autistic adults missing data on the PHQ-9 or DSM-5 GAD-A based on age, gender or AQ score.

As shown in table 6, the majority ( $43 \%$; $\mathrm{n}=98,4$ missing) of carers were mothers of an autistic adult, followed by partners or spouses $(23 \%)$. Carers' ages ranged from 21 to 78 years. The majority were female $(83 \%, \mathrm{n}=102)$, married $(66 \%)$ and living as a couple $(75 \%)$. Carers spent an average $63.4(\mathrm{SD}=57.80, \mathrm{n}=92)$ hours per week with the adult with autism, and the average age of the autistic adult cared for was 37.5 years (range 25-96 years, $\mathrm{SD}=13.21$ ). Most carer participants $(76 \%, \mathrm{n}=102)$ are linked to a selfreport or informant-report responses.

\section{FINDINGS TO DATE}

Using an inclusive research approach, the ALSAA has collected a detailed profile of the physical and mental health, productivity, well-being and health service use of autistic adults aged 25+ years, and their carers, living in Australia. There were high numbers of recruitment responses across the four time 1 survey populations (autistic and control adult self-report, informant report on autistic adults, carer self-report). The ALSAA time 1 data have been used in several studies of psychotropic medication use, ${ }^{75}$ leisure participation, ${ }^{76}$ ageing well, ${ }^{77}$ loneliness ${ }^{60}$ and employment. ${ }^{78}$ ALSAA and SASLA pooled data have been used to explore cognitive reappraisal, ${ }^{79}$ suicidal ideation, ${ }^{80}$ psychometric scale validation, ${ }^{64}$ and anxiety and depression. ${ }^{81}$ Several other outputs are in development, with focus on health service barriers and usage, caregivers, impact of diagnosis in adulthood, further scale validations, longitudinal analyses of loneliness, suicidal ideation, mental illness risk factors and other outputs, meeting the broad aim of the study to describe and compare the profiles of Australian autistic adults. Exploratory analyses have highlighted factors such as gender, education and employment that are potential confounders or covariates that need to be considered in future analyses.

\section{Participant characteristics}

A large number of females on the spectrum $(n=150)$ have participated in the ALSAA. This will provide an opportunity to explore the different expression of autism characteristics in females without intellectual disability. ${ }^{582}$ Similar to findings in other studies, ${ }^{10} 32$ self-reporting autistic adults had high rates of depression (52\%), anxiety (55\%) and a frequent history of psychiatric diagnosis $(89 \%)$, significantly more than the control sample. They were less likely to have a university education compared with the control sample.

\section{Questionnaire feedback and completion}

The length of the surveys may have led to some participants not completing following registration, or to missing data or only partial completions. Longer survey length has been linked to lower participation and completion of web-based surveys. ${ }^{83} 84$ Despite emphasising the ability to complete over several sessions, some participants commented that the survey was 'too long'. In contrast, survey length was not identified as an issue during pilot testing, time to complete was not related to survey satisfaction, and some participants appreciated the comprehensive nature of the surveys. Suggestions made in questionnaire feedback will be incorporated in the design of the 2-year follow-up. Follow-up design aims to reduce 
Table 5 Diagnostic characteristics

\begin{tabular}{|c|c|c|c|c|c|}
\hline Characteristic & $\begin{array}{l}\text { Autistic adults } \\
\text { self-report formally } \\
\text { diagnosed } \\
(n=221)\end{array}$ & $\begin{array}{l}\text { Autistic adults } \\
\text { self-report no } \\
\text { formal diagnosis } \\
(n=32)\end{array}$ & $\begin{array}{l}\text { Autistic adults } \\
\text { informant-report } \\
(n=42)\end{array}$ & $\begin{array}{l}\text { Carers } \\
(n=102)\end{array}$ & $\begin{array}{l}\text { Non-autistic self- } \\
\text { report } \\
(n=146)\end{array}$ \\
\hline AQ score mean (SD) & $87.6(10.3) n=210$ & $83.7(8.8)$ & $84.2(9.5) n=36$ & $\begin{array}{l}57.3(13.8) \\
n=60^{*}\end{array}$ & $55.1(11.9) n=137$ \\
\hline Above AQ cut-off (\%) & $203(97)$ & $31(97)$ & $35(97)$ & $18(30)$ & $30(22)$ \\
\hline \multicolumn{6}{|l|}{ †Autism diagnosis } \\
\hline $\begin{array}{l}\text { Autism spectrum } \\
\text { disorder/condition }\end{array}$ & $49(22)$ & - & $17(40)$ & - & - \\
\hline Asperger's syndrome & $130(59)$ & - & $11(26)$ & - & - \\
\hline Autistic disorder & $1(0)$ & - & $6(14)$ & - & - \\
\hline $\begin{array}{l}\text { 'High-functioning' } \\
\text { autism }\end{array}$ & $32(14)$ & - & $3(7)$ & - & - \\
\hline PDD-NOS & $1(0)$ & - & $1(2)$ & - & - \\
\hline Other & $6(3)$ & - & $2(5)$ & - & - \\
\hline No formal diagnosis & - & $32(100)$ & $1(2)$ & - & - \\
\hline $\begin{array}{l}\text { Age at diagnosis } \\
\text { mean (SD) }\end{array}$ & $35.3(15.1) n=217$ & - & $10.01(10.8) n=38$ & - & - \\
\hline $\begin{array}{l}\text { Years since diagnosis } \\
\text { mean (SD) }\end{array}$ & $6.9(8.3) n=217$ & - & $21.9(9.8) n=38$ & - & - \\
\hline $\begin{array}{l}\text { Intellectual disability } \\
(\%)\end{array}$ & $10(4)$ & $1(3)$ & $33(78)$ & $1(1)$ & $1(1)$ \\
\hline $\begin{array}{l}\text { Other developmental } \\
\text { disability (\%) }\end{array}$ & $6(3)$ & $2(6)$ & $9(21)$ & 0 & $1(1)$ \\
\hline \multicolumn{6}{|c|}{ ‡Other psychiatric diagnosis(\%) } \\
\hline Currently & 148 (69) $n=213$ & $13(42) n=31$ & $22(52)$ & $38(39) n=100$ & $29(21) n=140$ \\
\hline At any time & $191(89) n=213$ & $28(90) n=31$ & $31(74)$ & $68(68) n=100$ & $67(48) n=140$ \\
\hline $\begin{array}{l}\text { Depression (PHQ-9) } \\
\text { above cut-off (\%) }\end{array}$ & $103(52) n=199$ & $15(52) n=29$ & - & $28(29) n=97$ & 15 (12) $n=126$ \\
\hline $\begin{array}{l}\text { Anxiety (DSM-5 } \\
\text { GAD-A) above cut-off } \\
(\%)\end{array}$ & $115(56) n=206$ & $14(47) n=32$ & - & $27(27) n=99$ & $14(11) n=130$ \\
\hline
\end{tabular}

${ }^{*}$ Carers were asked to complete the $A Q$ scale only if they were a biological relative of the adult with autism.

†Autism diagnosis: Autistic adults (self-report, diagnosed), $n=220$.

$\ddagger$ Recording of a psychiatric diagnosis apart from autism spectrum conditions or intellectual disability.

AQ, Autism Quotient; GAD-A, Generalised Anxiety Disorder-Adult; PHQ-9, Patient Health Questionnaire-9.

assessment burden by reducing overall survey length and where appropriate prefilling repeated measures such as medical history. Additional areas of interest to the community will be explored including bullying, relationships and terms used to describe individuals with an autism spectrum diagnosis.

\section{Strengths and limitations}

An important strength of the study was the inclusive research approach using autistic advisors. Frequently missing from autism research and other longitudinal studies, an inclusive research protocol was developed for the ALSAA in consultation with autistic advisors (see table 1) and applied across all stages of the research process apart from the analysis. Inclusive research has been found to increase the likelihood that findings are translatable to the real world and focused on areas of need from community perspectives. ${ }^{44}$ Best-practise processes of conducting inclusive research with autistic adults are not well known, though more resources are becoming available. ${ }^{85}$ Although at times advisors' suggestions were in conflict with each other or beyond the scope of the study, as each advisor communicated individually with the research team there was no direct conflict between advisors. Feedback is given to advisors as to what advice has been used or not, and why. Although this potentially creates a power imbalance between the advisors and researchers, which is also inherent in an advisory process, to date there has been little feedback 


\begin{tabular}{|c|c|}
\hline Characteristic & Frequency/mean \\
\hline \multicolumn{2}{|l|}{ Relationship to autistic person (\%) } \\
\hline Mother & $42(43, n=98)$ \\
\hline Father & $10(10, n=98)$ \\
\hline Partner/spouse & $23(23, n=98)$ \\
\hline Sibling & $2(2, n=98)$ \\
\hline Child & $1(1, n=98)$ \\
\hline Carer-unspecified & $17(17, \mathrm{n}=98)$ \\
\hline Other & $3(3, n=98)$ \\
\hline $\begin{array}{l}\text { Age of autistic adult cared for mean } \\
\text { (SD) }\end{array}$ & $37.5(13.2)$ \\
\hline $\begin{array}{l}\text { Hours spent per week with autistic } \\
\text { adult mean (SD) }\end{array}$ & $63.4(57.8, n=92)$ \\
\hline
\end{tabular}

from advisors that was not able to be incorporated into the study processes or outputs. Ideally autistic adults and researchers would reach consensus in an open forum in person. Due to limitations with resourcing, geographical dispersion and majority of autistic advisors preferring to provide individual feedback in writing this was difficult to achieve. Moving forwards, we are considering implementing the 'Five-Finger Decision Method ${ }^{86}$ ' to ensure advisors input has been addressed. Little literature was available at the time of commencing the ALSAA and processes were developed iteratively based on understanding of partnering with autistic adults and guidance from our advisors. Our processes are largely consistent with recent guidelines now available, ${ }^{58}$ with deviation particularly relating to recruiting a balance of autistic community leaders and other autistic adults as advisors, a structured process for decision-making, and a lack of joint meetings/collaboration between advisors/a visioning exercise. We may benefit from again consulting with our advisors and seeing if there is interest in additional areas of involvement or additional peer-researched coauthored outputs. Growing relationships with advisors over time, and seeking feedback as to preferences for collaboration, has led to harmonious collaborative relationships between the ALSAA researchers and advisors, and will continue to evolve. An important consideration working with the advisors is the allowance of lead time and processing time, which has created difficulty for some student projects using ALSAA data. The ALSAA has further engaged peer researchers in some future outputs, including a study on autistic burnout, and the codevelopment of research grant applications for other projects. An additional strength of the ALSAA design is its consideration and partial overlap with several other national and international studies, for example, overlap with the Autism CRC SASLA longitudinal study allows for a lifespan approach to be taken including data from late adolescence. $^{79}$
The ALSAA baseline sample is reflective of the convenience sampling methodology used. Participants would appear non-representative of the Australian autism population given approximate postcode based above average socioeconomic status, a large female gender bias for both autistic adults and controls, and that most of the autistic sample $(85 \%)$ does not have an intellectual disability. Earlier epidemiological estimates of autism ${ }^{23}$ would suggest a larger proportion of people with a diagnosis of autism and intellectual disability and a much higher male to female ratio, though recent work questions these assumptions. ${ }^{287}$ Self-selection in online surveys can lead to a female gender bias, ${ }^{84}$ as also has been noted in other online autism research. ${ }^{88-90}$ Commonly reported as a 4 to 1 male bias, ${ }^{91}$ there is suggestion the true autism gender ratio may be closer to 3 to $1^{87}$ or lower ${ }^{82}$ as females with autism are at a higher risk of being underdiagnosed. ${ }^{3}$ Limiting recruitment to participants age 25 years or older, changing diagnostic practices ${ }^{92}$ in combination with the convenience sampling, has likely led to the large percentage of participants who had received their autism diagnosis in adulthood.

The majority of the current autistic sample does not have an intellectual disability. Significant barriers exist for people with intellectual disability to participate in a questionnaire-based study. ${ }^{93}$ An alternative to directly gathering information from adults on the spectrum with intellectual disability is the participation via an informant respondent. Although an informant respondent survey was made available for the ALSAA, and despite targeted recruitment efforts, there was low participation of people with intellectual disability.

A final limitation is the self-report questionnaire format of the ALSAA, particularly in relation to selfreported diagnosis of autism and comorbid conditions without clinical reassessment. Validating autism diagnosis though clinical reassessment would be a highly resource intensive exercise in a nationwide study in Australia and is beyond the resourcing available to this study. This is offset by gathering of additional information relating to autism diagnosis, including asking if the respondent has a diagnostic report, name and type of clinician providing diagnosis and $\mathrm{AQ}$ scores. These variables could be used to identify subsamples with stronger evidence of definite diagnosis of autism for separate analysis where necessary depending on the specific research question. Further, diagnostic reports from participants will be requested from participants at follow-up to confirm diagnosis. Gathering data from adults without a formal diagnosis is potentially a strength, given the 'lost generation' of autistic adults, ${ }^{3}$ significant barriers to diagnosis and accuracy issues with gold-standard autism assessment tools ${ }^{94}$; these participants could be overlooked in other studies though they represent an important hidden population group. The self-report format also prevents the quantification of participants' cognitive ability. The use of standardised mental health screening measures with clinical cut-offs gathers some information on co-occurring 
mental health conditions, though not as reliably as would be available via clinical interview. Conversely, communitybased sampling and use of self-report questionnaires does allow for gathering a reasonably large sample, offsetting the risk of including people with incorrect diagnostic data with increased statistical power and the potential severity bias of clinical samples. Limitations and strengths of the ALSAA study design will largely depend on the specific research question being explored, with sufficient sample size and data points to partial-out subgroups and non-matched controls or to account for common method variance as needed. Future researchers using the ALSAA dataset will need to consider balancing type 1 and type 2 errors. $^{73}$ A data governance structure has been established, with requests for ALSAA data requiring researchers to outline a statistical plan and data points they will be using, which will assist in managing any concerns of multiple statistical testing without correction. Any data requests will need to ensure that the hypotheses being tested do not duplicate use of data points in a way that would inflate the probability of type 1 errors.

\section{Implications}

There is a significant knowledge gap regarding the lives of autistic adults. The ALSAA has gathered a large comprehensive dataset relating to autistic adults and their carers, creating a platform for longitudinal follow-up and collaborative research. The ALSAA is an important resource for exploring multiple research questions regarding the lives of autistic adults. However, as with all longitudinal research caution is required in drawing firm conclusions from the ALSAA cohort in relation to the entire Australian autism population. Nevertheless, this cohort provides the first opportunity to explore longitudinal outcomes of Australian autistic adults. Having offline and informant versions of questionnaires, and questionnaires reviewed by autistic advisors, facilitates greater accessibility for participants on the spectrum and their carers.

The ALSAA study demonstrates a working approach to inclusive research with autistic advisors. The inclusive research protocol developed in this study provides a useful basis for other research endeavours. Following this protocol, future outputs such as peer-reviewed manuscripts, presentations and reports from the ALSAA will also be developed in consultation with autistic advisors or autistic peer researchers. Input from autistic advisors has already improved the quality and relevance of initial outputs, ${ }^{75}$ for example, identifying potential alternative interpretations of findings based on lived experiences, potential differences in autistic interpretations of scale items, and modifying the methodology in one instance ${ }^{60}$ changing a purely quantitative study into a mixedmethods design.

Some measures used in the ALSAA have not yet been validated with autistic adult samples (eg, DSM-5 GAD-A, Emotion Regulation Questionnaire, PHQ-15). The ALSAA provides the opportunity to explore the validity of these commonly used measures.

\section{Collaboration}

International studies involving autistic adults and utilising standardised measures are underway in the USA, Netherlands and UK (eg, SPARK http://sparkforautism. org, AASPIRE https://aaspire.org/, the Netherlands Autism Register https://www.nederlandsautismeregister. $\mathrm{nl}$ and the Adult Autism Spectrum Cohort-UK http:// research.ncl.ac.uk/adultautismspectrum/), though with less comprehensive ranges of measures. During development of the ALSAA, consultation with international groups occurred to ensure there were some synergies with these studies. Future international comparisons will be possible, including pooling of data. This will allow for the creation of much larger datasets and more in-depth understanding of life in adulthood for autistic people.

\section{Author affiliations}

${ }^{1}$ Department of Developmental Disability Neuropsychiatry (3DN), UNSW, Sydney, New South Wales, Australia

${ }^{2}$ Cooperative Research Centre for Living with Autism (Autism CRC), Brisbane, Queensland, Australia

${ }^{3}$ School of Health and Human Sciences, Southern Cross University, Gold Coast, Bilinga, Queensland, Australia

${ }^{4}$ Olga Tennison Autism Research Centre, School of Psychology and Public Health, La Trobe University, Bundoora, Victoria, Australia

${ }^{5}$ Stanford Autism Center, Division of Child and Adolescent Psychiatry, Department of Psychiatry and Behavioral Sciences, School of Medicine, Stanford University, Stanford, California, USA

${ }^{6}$ School of Occupational Therapy, Social Work and Speech Pathology, Curtin University, Perth, Western Australia, Australia

${ }^{7}$ School of Education and Communication, CHILD Programme, Institution of Disability Research, Jonkoping University, Jonkoping, Sweden

${ }^{8}$ Queensland Centre for Intellectual and Developmental Disability, Mater Research Institute - UQ, University of Queensland, South Brisbane, Queensland, Australia

${ }^{9}$ Health, Society \& Medicine Research Program, Social and Global Studies Centre, RMIT University, Melbourne, Victoria, Australia

Twitter Julian Trollor @3DN_UNSW

Acknowledgements The authors also acknowledge the valuable contribution of the Research Advisory Network members Matthew Bennett, Bob Boyce, Jen Harland, Julianne Higgins, Gabriel Nakhel, Joanne Mahony, Andrea Michael, Cheryl Strangio and Chris Tanner whose insight and recommendations shaped this work. We also gratefully acknowledge the cooperation and participation of all participants on the autism spectrum and their carers involved in this study.

Contributors Manuscript drafting was jointly led by SA and K-RF. All authors (YI(J)H, ALR, MU, LPL, RYC, TF, MF, NGL, AU and JT) had a role in the conduct of the research, interpretation of results, drafting of manuscript and critical review of the manuscript. SA, K-RF, YI(J)H, ALR, MU and JT conducted data analysis and reporting. K-RF, YI(J)H, ALR, MU, RYC, TF, MF, NGL, AU and JT were involved in the conceptualisation of the study.

Funding This work was supported by the Cooperative Research Centre for Living with Autism (Autism CRC), established and supported under the Australian Government's Cooperative Research Centres Program.

Competing interests None declared.

Patient consent for publication Not required.

Ethics approval The study was approved by the Human Research Ethics Committee at UNSW Sydney, Australia (No. HC15001).

Provenance and peer review Not commissioned; externally peer reviewed.

Data availability statement Data may be obtained from a third party and are not publicly available.

Open access This is an open access article distributed in accordance with the Creative Commons Attribution Non Commercial (CC BY-NC 4.0) license, which permits others to distribute, remix, adapt, build upon this work non-commercially, 
and license their derivative works on different terms, provided the original work is properly cited, appropriate credit is given, any changes made indicated, and the use is non-commercial. See: http://creativecommons.org/licenses/by-nc/4.0/.

\section{ORCID iDs}

Samuel Arnold http://orcid.org/0000-0003-2900-223X

Julian Trollor http://orcid.org/0000-0002-7685-2977

\section{REFERENCES}

1 Seltzer MM, Shattuck P, Abbeduto L, et al. Trajectory of development in adolescents and adults with autism. Ment Retard Dev Disabil Res Rev 2004;10:234-47.

2 Brugha TS, Spiers N, Bankart J, et al. Epidemiology of autism in adults across age groups and ability levels. Br J Psychiatry 2016;209:498-503. en

3 Lai M-C, Baron-Cohen S. Identifying the lost generation of adults with autism spectrum conditions. Lancet Psychiatry 2015;2:1013-27.

4 Van Wijngaarden-Cremers PJM, van Eeten E, Groen WB, et al. Gender and age differences in the core triad of impairments in autism spectrum disorders: a systematic review and meta-analysis. J Autism Dev Disord 2014;44:627-35.

5 Bargiela S, Steward R, Mandy W. The experiences of Latediagnosed women with autism spectrum conditions: an investigation of the female autism phenotype. J Autism Dev Disord 2016;46:3281-94. en

6 Jang J, Matson JL, Adams HL, et al. What are the ages of persons studied in autism research: a 20 -year review. Res Autism Spectr Disord 2014;8:1756-60.

7 Howlin P, Moss P. Adults with autism spectrum disorders. Can J Psychiatry 2012;57:275-83.

8 Nicolaidis C. Autism in Adulthood: The New Home for Our Emerging Field. Autism in Adulthood 2019;1:1-3.

9 Michael C. Why we need research about autism and ageing. Autism 2016;20:515-6.

10 Howlin P, Magiati I. Autism spectrum disorder: outcomes in adulthood. Curr Opin Psychiatry 2017;30:69-76. en.

11 Cashin A, Buckley T, Trollor JN, et al. A scoping review of what is known of the physical health of adults with autism spectrum disorder. J Intellect Disabil 2018;22:96-108.

12 Croen LA, Zerbo O, Qian Y, et al. The health status of adults on the autism spectrum. Autism 2015;19:814-23.

13 Jones KB, Cottle K, Bakian A, et al. A description of medical conditions in adults with autism spectrum disorder: a follow-up of the 1980s Utah/UCLA autism epidemiologic study. Autism 2016;20:551-61.

14 Weiss JA, Burnham Riosa P, Riosa PB. Thriving in youth with autism spectrum disorder and intellectual disability. J Autism Dev Disord 2015:45:2474-86

15 Buck TR, Viskochil J, Farley M, et al. Psychiatric comorbidity and medication use in adults with autism spectrum disorder. J Autism Dev Disord 2014;44:3063-71.

16 Hedley D, Uljarević M, Wilmot M, et al. Understanding depression and thoughts of self-harm in autism: a potential mechanism involving loneliness. Res Autism Spectr Disord 2018;46:1-7.

17 Lever AG, Geurts HM. Psychiatric co-occurring symptoms and disorders in young, middle-aged, and older adults with autism spectrum disorder. J Autism Dev Disord 2016;46:1916-30.

18 Matson JL, Williams LW. Depression and mood disorders among persons with autism spectrum disorders. Res Dev Disabil 2014;35:2003-7.

19 Moss P, Howlin P, Savage S, et al. Self and informant reports of mental health difficulties among adults with autism findings from a long-term follow-up study. Autism 2015;19:832-41.

20 Hedley D, Uljarević M, Wilmot M, et al. Brief report: social support, depression and suicidal ideation in adults with autism spectrum disorder. J Autism Dev Disord 2017:47:3669-77.

21 Hirvikoski T, Mittendorfer-Rutz E, Boman M, et al. Premature mortality in autism spectrum disorder. Br J Psychiatry 2016;208:232-8.

22 Matson JL, Nebel-Schwalm MS. Comorbid psychopathology with autism spectrum disorder in children: an overview. Res Dev Disabil 2007:28:341-52.

23 Fombonne E. Epidemiological surveys of autism and other pervasive developmental disorders: an update. J Autism Dev Disord 2003;33:365-82.

24 La Malfa G, Lassi S, Bertelli M, et al. Autism and intellectual disability: a study of prevalence on a sample of the Italian population. J Intellect Disabil Res 2004;48:262-7.
25 Matson JL, Shoemaker M. Intellectual disability and its relationship to autism spectrum disorders. Res Dev Disabil 2009;30:1107-14.

26 Dawson M, Soulières I, Ann Gernsbacher M, et al. The level and nature of autistic intelligence. Psychol Sci 2007;18:657-62.

27 Howlin P, Arciuli J, Begeer S, et al. Research on adults with autism spectrum disorder: roundtable report. J Intellect Disabil Res 2015;40:388-93.

28 Hedley D, Uljarević M, Cameron L, et al. Employment programmes and interventions targeting adults with autism spectrum disorder: a systematic review of the literature. Autism 2017;21:929-41.

29 Gray KM, Piccinin A, Keating CM, et al. Outcomes in young adulthood: are we achieving community participation and inclusion? J Intellect Disabil Res 2014;58:734-45.

30 Henninger NA, Taylor JL. Outcomes in adults with autism spectrum disorders: a historical perspective. Autism 2013;17:103-16.

31 Howlin P, Moss P, Savage S, et al. Social outcomes in mid- to later adulthood among individuals diagnosed with autism and average nonverbal IQ as children. J Am Acad Child Adolesc Psychiatry 2013;52:572-81.

32 Magiati I, Tay XW, Howlin P, Cognitive HP. Cognitive, language, social and behavioural outcomes in adults with autism spectrum disorders: a systematic review of longitudinal follow-up studies in adulthood. Clin Psychol Rev 2014;34:73-86.

33 Marriage S, Wolverton A, Marriage K. Autism spectrum disorder grown up: a chart review of adult functioning. $J$ Can Acad Child Adolesc Psychiatry 2009;18:322-8.

34 Levy A, Perry A. Outcomes in adolescents and adults with autism: a review of the literature. Res Autism Spectr Disord 2011;5:1271-82.

35 Seltzer MM, Krauss MW, Shattuck PT, et al. The symptoms of autism spectrum disorders in adolescence and adulthood. J Autism Dev Disord 2003;33:565-81.

36 Shattuck PT, Seltzer MM, Greenberg JS, et al. Change in autism symptoms and maladaptive behaviors in adolescents and adults with an autism spectrum disorder. J Autism Dev Disord 2007;37:1735-47.

37 Happé F, Charlton RA. Aging in autism spectrum disorders: a minireview. Gerontology 2012;58:70-8.

38 Totsika V, Felce D, Kerr M, et al. Behavior problems, psychiatric symptoms, and quality of life for older adults with intellectual disability with and without autism. J Autism Dev Disord 2010;40:1171-8.

39 Khanna R, Jariwala-Parikh K, West-Strum D, Mahabaleshwarkar $\mathrm{R}$, et al. Health-Related quality of life and its determinants among adults with autism. Res Autism Spectr Disord 2014;8:157-67.

40 Pellicano E, Dinsmore A, Charman T. What should autism research focus upon? community views and priorities from the United Kingdom. Autism 2014;18:756-70.

41 Nicolaidis $\mathrm{C}$. What can physicians learn from the neurodiversity movement? Virtual Mentor 2012:14:503-10.

42 Nicolaidis C, Raymaker D, McDonald K, Dern S, et al. Comparison of healthcare experiences in autistic and non-autistic adults: a cross-sectional online survey facilitated by an academic-community partnership. J Gen Intern Med 2013;28:761-9.

43 Kapp SK, Steward R, Crane L, et al. 'People should be allowed to do what they like': Autistic adults' views and experiences of stimming. Autism 2019;23:1782-92.

44 Walmsley J, Johnson K. Inclusive research with people with learning disabilities: past, present and futures. London and New York: Jessica Kingsley Publishers, 2003.

45 Bigby C, Frawley P, Ramcharan P. Conceptualizing inclusive research with people with intellectual disability. J Appl Res Intellect Disabil 2014:27:3-12.

46 Bagatell N. From cure to community: transforming notions of autism. Ethos 2010;38:33-55.

47 Milton D, Bracher M. Autistics speak but are they heard? Med Sociology Online 2013;7:61-9.

48 Nind M, Vinha H. Doing research inclusively: bridges to multiple possibilities in inclusive research. Br J Learn Disabil 2014;42:102-9.

49 Gatfield O, Mangan C, Haar T, et al. Research priorities survey report 2016. Brisbane, Queensland, Australia: Cooperative Research Centre for Living with Autism, 2016.

50 Gotham K, Marvin AR, Taylor JL, et al. Characterizing the daily life, needs, and priorities of adults with autism spectrum disorder from interactive autism network data. Autism 2015;19:794-804.

51 McConachie H, Mason D, Parr JR, et al. Enhancing the validity of a quality of life measure for autistic people. J Autism Dev Disord $2018 ; ; 48: 1596-611$.

52 Seltzer MM, Greenberg JS, Taylor JL, et al. Adolescents and adults with autism spectrum disorders. In: Amaral DG, Dawson G, Geschwind D, et al, eds. Autism spectrum disorders. New York: Oxford University Press, 2011. 
53 Lawson L, Hascheck A, Richdale AL. SASLA snapshot: study of Australian School-Leavers with autism - baseline profile. Melbourne, Australia: Olga Tennison Autism Research Centre (OTARC), La Trobe University, 2018.

54 Haas K, Costley D, Falkmer M, et al. Factors influencing the research participation of adults with autism spectrum disorders. $J$ Autism Dev Disord 2016 ;;46:1793-805.

55 Walmsley J. Normalisation, emancipatory research and inclusive research in learning disability. Disabil Soc 2001;16:187-205.

56 AutismCRC. Inclusive research practive guide and checklists for autism research: version 2. Brisbane, Queensland: Autism CRC Ltd, 2016.

57 Nicolaidis C, Raymaker D, Katz M, et al. Community-Based participatory research to adapt health measures for use by people with developmental disabilities. Prog Community Health Partnersh 2015;9:141-3

58 Nicolaidis C, Raymaker D, Kapp SK, et al. The AASPIRE practicebased guidelines for the inclusion of autistic adults in research as co-researchers and study participants. Autism 2019;23:2007-19.

59 Jivraj J, Sacrey L-A, Newton A, et al. Assessing the influence of researcher-partner involvement on the process and outcomes of participatory research in autism spectrum disorder and neurodevelopmental disorders: a scoping review. Autism 2014;18:782-93.

60 Ee D, Hwang Ye In (Jane), Reppermund S, et al. Loneliness in adults on the autism spectrum. Autism in Adulthood 2019;1:182-93.

61 Kroenke K, Spitzer RL, Williams JB. The PHQ-9: validity of a brief depression severity measure. J Gen Intern Med 2001;16:606-13.

62 Khanna R, Jariwala K, West-Strum D. Validity and reliability of the medical outcomes study short-form health survey version 2 (SF12v2) among adults with autism. Res Dev Disabil 2015;43-44:51-60.

63 Barrett SL, Uljarević M, Baker EK, et al. The adult repetitive behaviours Questionnaire-2 (RBQ-2A): a self-report measure of restricted and repetitive behaviours. J Autism Dev Disord 2015;45:3680-92.

64 Arnold SRC, Uljarević M, Hwang YI, Hwang J, et al. Brief report: psychometric properties of the patient health Questionaire-9 (PHQ9) in autistic adults. J Autism Dev Disord 2019;47:1-9.

65 Whitehouse AJO, Bishop DVM. Communication checklist - adult. London: The Psychological Corporation, 2009.

66 (AlHW) AloHaW. Autism in Australia. Web report. Canberra, Australia: AlHW, 2017.

67 Hoekstra RA, Vinkhuyzen AAE, Wheelwright S, et al. The construction and validation of an abridged version of the autism-spectrum quotient (AQ-Short). J Autism Dev Disord 2011:41:589-96.

68 Lundqvist $\mathrm{L}-\mathrm{O}$, Lindner $\mathrm{H}$. Is the autism-spectrum quotient a valid measure of traits associated with the autism spectrum? A Rasch validation in adults with and without autism spectrum disorders. $J$ Autism Dev Disord 2017;47:2080-91.

69 Eriksson JM, Andersen LM, Bejerot S. RAADS-14 screen: validity of a screening tool for autism spectrum disorder in an adult psychiatric population. Mol Autism 2013;4:49.

70 Evans E, Trollor J, Davis A, et al. Mental health and carer support in elders with ID. Report to ageing, disability and home care, department of family and community services, NSW Australia. Sydney, Australia: Department of Developmental Disability Neuropsychiatry, UNSW Sydney, 2015.

71 StataCorp. Stata statistical software: release 14. College Station, TX: StataCorp LP, 2015.

72 IBM_Corp. Ibm SPSS statistics for windows, version 22.0. Armonk, NY: IBM Corp, 2013.

73 Streiner DL, Norman GR. Correction for multiple testing: is there a resolution? Chest 2011;140:16-18.

74 Beesdo-Baum K, Klotsche J, Knappe S, et al. Psychometric properties of the dimensional anxiety scales for DSM-V in an unselected sample of German treatment seeking patients. Depress Anxiety 2012;29:1014-24.

75 Cvejic RC, Arnold SRC, Foley K-R, et al. Neuropsychiatric profile and psychotropic medication use in adults with autism spectrum disorder: results from the Australian longitudinal study of adults with autism. BJPsych Open 2018;4:461-6.

76 Stacey T-L, Froude EH, Trollor J, et al. Leisure participation and satisfaction in autistic adults and neurotypical adults. Autism 2018.

77 Hwang YI, Foley K-R, Trollor JN. Aging well on the autism spectrum: an examination of the dominant model of successful aging. $J$ Autism Dev Disord 2018;31.

78 Harsanyi M, Foley K-R, Froude E. Employment profiles of autistic adults in Australia, 2019.

79 Cai RY, Richdale AL, Foley K-R, et al. Brief report: cross-sectional interactions between expressive suppression and cognitive reappraisal and its relationship with depressive symptoms in autism spectrum disorder. Res Autism Spectr Disord 2018;45:1-8.

80 Hedley D, Uljarević M, Foley K-R, et al. Risk and protective factors underlying depression and suicidal ideation in autism spectrum disorder. Depress Anxiety 2018;35:648-57.

81 Uljarević M, Hedley D, Rose-Foley K, Foley K-R, et al. Anxiety and depression from adolescence to old age in autism spectrum disorder. J Autism Dev Disord 2019;38.

82 Baldwin S, Costley D. The experiences and needs of female adults with high-functioning autism spectrum disorder. Autism 2016;20:483-95.

83 Fan W, Yan Z. Factors affecting response rates of the web survey: a systematic review. Comput Human Behav 2010;26:132-9.

84 Guo X, Vittinghoff E, Olgin JE, et al. Volunteer participation in the health eHeart study: a comparison with the US population. Sci Rep 2017;7:1956. En.

85 Fletcher-Watson S, Adams J, Brook K, et al. Making the future together: shaping autism research through meaningful participation Autism 2019;23:943-53.

86 Nicolaidis C, Raymaker D, McDonald K, et al. Collaboration strategies in nontraditional community-based participatory research partnerships: lessons from an academic-community partnership with autistic self-advocates. Prog Community Health Partnersh 2011;5:143-50.

87 Loomes R, Hull L, Mandy WPL. What is the male-to-female ratio in autism spectrum disorder? A systematic review and meta-analysis. J Am Acad Child Adolesc Psychiatry 2017;56:466-74.

88 Gilmour L, Schalomon PM, Smith V. Sexuality in a community based sample of adults with autism spectrum disorder. Res Autism Spectr Disord 2012;6:313-8.

89 Kapp SK, Gillespie-Lynch K, Sherman LE, et al. Deficit, difference, or both? autism and neurodiversity. Dev Psychol 2013;49:59-71. en

90 Nicolaidis C, Raymaker D, McDonald K, et al. Comparison of healthcare experiences in autistic and non-autistic adults: a crosssectional online survey facilitated by an academic-community partnership. J Gen Intern Med 2013;28:761-9.

91 Whiteley P, Todd L, Carr K, et al. Gender ratios in autism, Asperger syndrome and autism spectrum disorder. London: Autism Insights, 2010: 2. 17.English

92 Whitehouse AJO, Cooper MN, Bebbington K, et al. Evidence of a reduction over time in the behavioral severity of autistic disorder diagnoses. Autism Research 2017;10:179-87.

93 Chadwick D, Wesson C, Fullwood C. Internet access by people with intellectual disabilities: inequalities and opportunities. Future Internet 2013;5:376-97.

94 Fusar-Poli L, Brondino N, Rocchetti M, et al. Diagnosing ASD in adults without ID: accuracy of the ADOS-2 and the ADI-R. J Autism Dev Disord 2017;47:3370-9.

95 Robertson AE, Simmons DR. The relationship between sensory sensitivity and autistic traits in the general population. J Autism Dev Disord 2013;43:775-84.

96 Carleton RN, Norton MAPJ, Asmundson GJG. Fearing the unknown: a short version of the intolerance of uncertainty scale. $J$ Anxiety Disord 2007;21:105-17.

97 Spitzer RL, Williams JB, Kroenke K, et al. Utility of a new procedure for diagnosing mental disorders in primary care. The PRIME-MD 1000 study. JAMA 1994;272:1749-56.

98 Ware J, Kosinski M, Keller SD. A 12-Item short-form health survey: construction of scales and preliminary tests of reliability and validity. Med Care 1996;34:220.

99 Sletten DM, Suarez GA, Low PA, et al. Compass 31: a refined and abbreviated composite autonomic symptom score. Mayo Clin Proc 2012;87:1196-201

100 Buysse DJ, Reynolds CF, Monk TH, et al. The Pittsburgh sleep quality index: a new instrument for psychiatric practice and research. Psychiatry Res 1989;28:193-213.

101 Gradisar M, Lack L, Richards H, et al. The Flinders fatigue scale: preliminary psychometric properties and clinical sensitivity of a new scale for measuring daytime fatigue associated with insomnia. $J$ Clin Sleep Med 2007:3:722-8.

102 Craske MG, Wittchen H-U, Bogels S, et al. Severity measure for Generalized Anxiety Disorder - adult. American Psychiatric Association, 2013.

103 Tennant R, Hiller L, Fishwick R, et al. The Warwick-Edinburgh mental well-being scale (WEMWBS): development and UK validation. Health Qual Life Outcomes 2007;5:63.

104 Chen G, Gully SM, Eden D. Validation of a new general self-efficacy scale. Organ Res Methods 2001;4:62-83.

105 Gross JJ, John OP. Individual differences in two emotion regulation processes: implications for affect, relationships, and well-being. $J$ Pers Soc Psychol 2003;85:348-62. 
106 Mohr C, Tonge BJ, Einfeld SL, et al. The developmental behaviour checklist for adults (DBC-A) revised. Sydney, Australia: University of Sydney and Monash University, 2011.

107 Carver CS. You want to measure coping but your protocol' too long: Consider the brief cope. Int J Behav Med 1997;4:92-100.

108 Crook TH, Feher EP, Larrabee GJ. Assessment of memory complaint in age-associated memory impairment: the MAC-Q. Int Psychogeriatr 1992;4:165-76.

109 Hays R, DiMatteo MR. A short-form measure of loneliness. J Pers Assess 1987;51:69-81.

110 Sarason IG, Levine HM, Basham RB, et al. Assessing social support: the social support questionnaire. J Pers Soc Psychol 1983;44:127-39.

111 The WHOQOL Group. The World Health Organization Quality of Life assessment (WHOQOL)-BREF quality of life assessment. Psychological Medicine 1995;28:551-5.

112 Maenner MJ, Smith LE, Hong J, et al. Evaluation of an activities of daily living scale for adolescents and adults with developmental disabilities. Disabil Health J 2013;6:8-17.

113 Luciano JV, Ayuso-Mateos JL, Aguado J, et al. The 12-Item World Health organization disability assessment schedule II (WHO-DAS II) a nonparametric item response analysis. BMC Med Res Methodol 2010;10:1-9.
114 Taylor JL, Seltzer MM. Developing a vocational index for adults with autism spectrum disorders. J Autism Dev Disord 2012;42:2669-79.

115 Beard JG, Ragheb MG. Measuring leisure satisfaction. J Leis Res 1980;12:20-33.

116 Chisholm D, Knapp MRJ, Knudsen HC, et al. Client sociodemographic and service receipt inventory - European version: development of an instrument for international research. $\mathrm{Br} J$ Psychiatry 2000;177:s28-33.

117 Bengston VL, Allen KR. The life course perspective applied to families overtime. In: Boss P, Doherty W, LaRossa R, eds. Sourcebook of family theories and methods: a contextual approach New York: Plenum Press, 1993: 469-98.

118 McCarron M, Gill M, Lawlor B, et al. A pilot study of the reliability and validity of the Caregiver Activity Survey - Intellectual Disability (CAS-ID). J Intellect Disabil Res 2002;46:605-12.

119 Bédard M, Molloy DW, Squire L, et al. The Zarit burden interview: a new short version and screening version. Gerontologist 2001;41:652-7.

120 Alliance NRH. The litte book of rural health numbers: author, 2015. Available: https://www.ruralhealth.org.au/book/little-book-ruralhealth-numbers 